\title{
Saturn's polar ionospheric flows and their relation to the main auroral oval
}

\author{
S. W. H. Cowley ${ }^{1}$, E. J. Bunce ${ }^{1}$, and R. Prangé en $^{2}$ \\ ${ }^{1}$ Department of Physics \& Astronomy, University of Leicester, Leicester LE1 7RH, UK \\ ${ }^{2}$ LESIA, Observatoire de Paris, 92195 Meudon, France
}

Received: 7 April 2003 - Revised: 12 July 2003 - Accepted: 22 July 2003 - Published: 2 April 2004

\begin{abstract}
We consider the flows and currents in Saturn's polar ionosphere which are implied by a three-component picture of large-scale magnetospheric flow driven both by planetary rotation and the solar wind interaction. With increasing radial distance in the equatorial plane, these components consist of a region dominated by planetary rotation where planetary plasma sub-corotates on closed field lines, a surrounding region where planetary plasma is lost down the dusk tail by the stretching out of closed field lines followed by plasmoid formation and pinch-off, as first described for Jupiter by Vasyliunas, and an outer region driven by the interaction with the solar wind, specifically by reconnection at the dayside magnetopause and in the dawn tail, first discussed for Earth by Dungey. The sub-corotating flow on closed field lines in the dayside magnetosphere is constrained by Voyager plasma observations, showing that the plasma angular velocity falls to around half of rigid corotation in the outer magnetosphere, possibly increasing somewhat near the dayside magnetopause, while here we provide theoretical arguments which indicate that the flow should drop to considerably smaller values on open field lines in the polar cap. The implied ionospheric current system requires a four-ring pattern of field-aligned currents, with distributed downward currents on open field lines in the polar cap, a narrow ring of upward current near the boundary of open and closed field lines, and regions of distributed downward and upward current on closed field lines at lower latitudes associated with the transfer of angular momentum from the planetary atmosphere to the sub-corotating planetary magnetospheric plasma. Recent work has shown that the upward current associated with sub-corotation is not sufficiently intense to produce significant auroral acceleration and emission. Here we suggest that the observed auroral oval at Saturn instead corresponds to the ring of upward current bounding the region of open and closed field lines. Estimates indicate that auroras of brightness from a few $\mathrm{kR}$ to a few tens of $\mathrm{kR}$ can be produced by
\end{abstract}

Correspondence to: S. W. H. Cowley

(swhc1@ion.le.ac.uk) precipitating accelerated magnetospheric electrons of a few $\mathrm{keV}$ to a few tens of keV energy, if the current flows in a region which is sufficiently narrow, of the order of or less than $\sim 1000 \mathrm{~km}\left(\sim 1^{\circ}\right.$ latitude $)$ wide. Arguments are also given which indicate that the auroras should typically be significantly brighter on the dawn side of the oval than at dusk, by roughly an order of magnitude, and should be displaced somewhat towards dawn by the down-tail outflow at dusk associated with the Vasyliunas cycle. Model estimates are found to be in good agreement with data derived from high quality images newly obtained using the Space Telescope Imaging Spectrograph on the Hubble Space Telescope, both in regard to physical parameters, as well as local time effects. The implication of this picture is that the form, position, and brightness of Saturn's main auroral oval provide remote diagnostics of the magnetospheric interaction with the solar wind, including dynamics associated with magnetopause and tail plasma interaction processes.

Key words. Magnetospheric physics (auroral phenomena, magnetosphere-ionosphere interactions, solar windmagnetosphere interactions)

\section{Introduction}

First indications of the existence of UV auroras in Saturn's polar regions were obtained by the UV photometer on board the Pioneer-11 spacecraft during its flyby of the planet in 1979 (Judge et al., 1980), together with remote observations by the IUE spacecraft (Clarke et al., 1981; McGrath and Clarke, 1992). Unambiguous detections were obtained subsequently by the UVS instruments on the two Voyager spacecraft during their flybys in 1980 and 1981 (Broadfoot et al., 1981; Sandel and Broadfoot, 1981; Sandel et al., 1982; Shemansky and Ajello, 1983). The latter observations indicated that the auroras are due to $\mathrm{keV}$ electron impact on molecular hydrogen, and take the form of bands of emission around each pole which are essentially continuous in local 


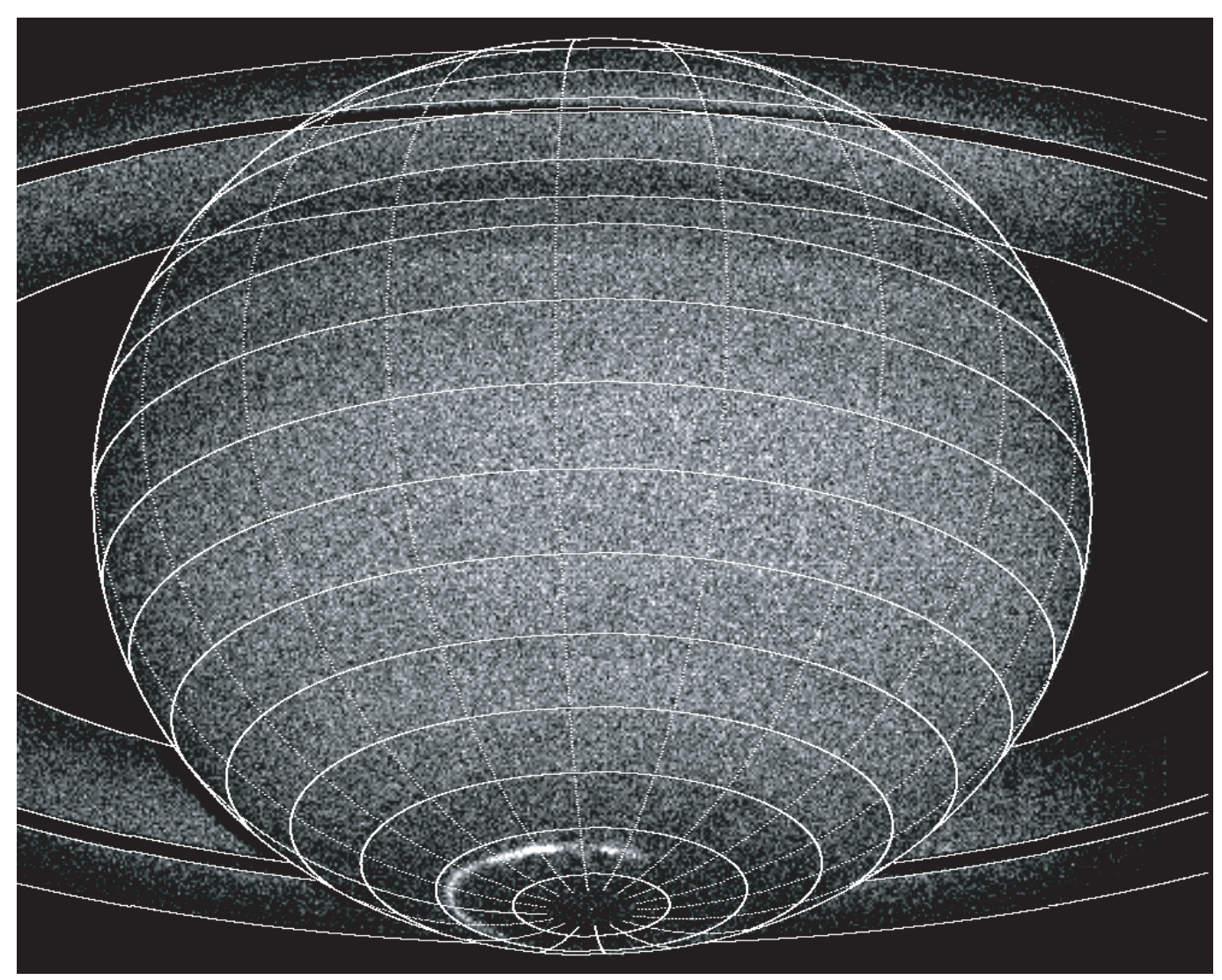

Fig. 1. UV image of Saturn obtained using the Space Telescope Imaging Spectrograph (STIS) instrument on the Hubble Space Telescope at 10:00 UT (start time) on 8 December 2000. The exposure time was $480 \mathrm{~s}$, during which Saturn rotated by $\sim 5^{\circ}$. The $\mathrm{H}_{2} \mathrm{Werner}$ and Lyman bands above $\sim 130 \mathrm{~nm}$ were isolated by using filter SRF2. An oblate spheroid and a grid of coordinates have been over-plotted, the latter at latitude steps of $10^{\circ}$ and longitude steps of $20^{\circ}$. The sub-Earth point is at longitude $\mathrm{SEP}=283^{\circ}$.

time, and also continuous at a given local time with respect to the spin phase of the planet. The brightness in the prenoon sector, however, was found to be modulated during the planet's rotation (Sandel and Broadfoot, 1981; Sandel et al., 1982), approximately in phase with a similar modulation of the Saturn kilometric radiation (SKR) (Gurnett et al., 1981; Kaiser et al., 1984). Overall, the Voyager observations indicated that the brightness of the auroras is very variable on time scales from tens of minutes to hours, over the range from $\sim 1$ to $\sim 100 \mathrm{kR}$ of total $\mathrm{H}_{2}$ emission. Although precise determination of the latitude distribution was difficult to obtain from the UVS data, observations of the Southern Hemisphere aurora close to the dusk meridian obtained by Voyager-1 near periapsis indicated that the emissions were confined to co-latitudes between $\sim 9.5^{\circ}$ and $\sim 12^{\circ}$ (Sandel and Broadfoot, 1981). More recently, images of Saturn's UV auroras have also been obtained by the Hubble Space Telescope (HST). The first of these, obtained by Gérard et al. (1995), using the Faint Object Camera (FOC), suggested the presence of an auroral oval around the northern pole in the co-latitude range $\sim 10^{\circ}-15^{\circ}$, with an average brightness of $\sim 150 \mathrm{kR}$ of total $\mathrm{H}_{2}$ emission. A few images have also been presented by Trauger et al. (1998) using the Wide Field and Planetary Camera 2 (WFPC2). These show the presence of oval emissions above a $\sim 5 \mathrm{kR}$ threshold over a wide range of local times, but strongly peaking in brightness between $\sim 7-9 \mathrm{~h} \mathrm{LT}$ in the pre-noon sector at co-latitudes between $\sim 13^{\circ}$ and $\sim 15^{\circ}$. Again, however, images which were well separated in UT show considerable variation in brightness, from no emission at all above the $\sim 5 \mathrm{kR}$ threshold, to emissions peaking (in the pre-noon sector) at $\sim 100 \mathrm{kR}$. When UV emission is present, however, its location is generally found to lie within the envelope of the principal source region of SKR (Lecacheux and Genova, 1983; Galopeau et al., 1995).

Most recently, UV images of Saturn's aurora have also been obtained using the Space Telescope Imaging Spectrograph (STIS) instrument on the HST. STIS combines a spatial resolution almost equal to that of the FOC $(0.0244 \mathrm{arcs}$ square pixels and 2-3 pixel-FWHM point spread function (PSF)), with a sensitivity which is a few times greater than that of the WFPC2, thus providing the most complete picture yet obtained of Saturn's auroral morphology. Two such images were obtained on 7 and 8 December 2000, when the southern auroral oval was completely visible from the Earth due to the large tilt of Saturn's rotation axis, while the northern oval was hidden on the far side, beyond Saturn's limb. In Fig. 1 we show the image obtained on 8 December 2000 , together with a superposed latitude-longitude grid. 
Table 1. Summary of Saturn's auroral oval characteristics obtained from the HST-STIS image obtained on 8 December 2000 and shown in Fig. 1 (except as indicated).

\begin{tabular}{cccccc}
\hline & Dawn & $\begin{array}{c}\text { Pre-noon } \\
\text { maximum }\end{array}$ & Afternoon & Dusk & Pre-midnight \\
\hline Co-latitude $(\mathrm{deg})$ & $18-18.5$ & $14-15.5$ & $12-13$ & $14-15$ (7 Dec.) & $>15$ \\
\hline Width $(\mathrm{km})$ & $500-700$ & $270-340$ & $700-950$ & $\gg 1000$ & - \\
\hline Brightness $(\mathrm{kR})$ & $20-25$ & $45-60$ & $3-12$ & $\leq 1.5-3$ & $3-6$ \\
\hline Electron energy flux $\left(\mathrm{mW} \mathrm{m}^{-2}\right)$ & $1.9-3.2$ & $4.2-7.7$ & $0.28-1.5$ & $\leq 0.14-0.38$ & $0.28-0.77$ \\
\hline
\end{tabular}

This shows the presence of a "discrete" auroral oval which extends continuously from the midnight sector via dawn into the post-noon hours, though varying strongly in brightness with local time. Analysis to be presented elsewhere shows that the brightness was $\sim 20-25 \mathrm{kR}$ at dawn, and peaked at $\sim 45 \mathrm{kR}$ in the pre-noon sector (09:30-11 LT), with a localised maximum of $\sim 60 \mathrm{kR}$. However, the emission generally fell below the instrument sensitivity threshold $(\sim 1.5 \mathrm{kR})$ in the late afternoon and dusk sector, while re-appearing at a detectable level at $\sim 20 \mathrm{LT}$ and increasing to $\sim 3-6 \mathrm{kR}$ in the pre-midnight sector. The co-latitude of the emission also clearly varied with local time, lying at $\sim 18^{\circ}-18.5^{\circ}$ at dawn, while decreasing to $\sim 14^{\circ}-15.5^{\circ}$ at noon, and to $\sim 12^{\circ}-13^{\circ}$ in the early afternoon. The latitudinal distribution is difficult to determine in the pre-midnight sector, but was present at co-latitudes greater than $\sim 15^{\circ}$. The latitudinal width of the emission, de-convolved from the PSF, varied from a minimum value of $\sim 270-340 \mathrm{~km}$ FWHM in the brightest prenoon sector (the error bar being due to uncertainties in the PSF), to $\sim 500-700 \mathrm{~km}$ at dawn, and $\sim 700-950 \mathrm{~km}$ in the early afternoon. The image obtained on 7 December (to be presented elsewhere) shows similar characteristics, though the overall brightness was larger by $\sim 50 \%$. This image also reveals the presence of multiple auroral branches at $\sim 14^{\circ}-15^{\circ}$ co-latitude in the dusk sector, with brightnesses of $\sim 3-8 \mathrm{kR}$ and an overall latitudinal thickness of several thousand $\mathrm{km}$. Detailed examination shows that vestiges of these emissions can also be discerned near the instrument threshold in the image obtained on 8 December shown in Fig. 1. A summary of the properties of the emissions observed in the latter image is given in Table 1, for later comparison with theoretical estimates. The values of the precipitating electron energy flux shown in the bottom row of the table were derived from considerations to be discussed in Sect. 4 below.

This paper addresses the issue of the physical origins of these auroras. Their discrete nature observed in the STIS images suggests that they are produced by downwardaccelerated precipitating electrons in regions of upwarddirected field-aligned current flowing between the magnetosphere and the high-latitude ionosphere. We note that the existence of such currents has previously been inferred from analysis of Voyager flyby magnetic field data by Connerney et al. (1983) (see also Bunce et al., 2003). With regard to the physical origins of the currents, two basic possibilities can be considered, as will now be briefly discussed. The first possibility is that they are associated with the current system that transfers angular momentum from the atmosphere of the rapidly-spinning planet to the sub-corotating equatorial magnetospheric plasma (Hill, 1979; Vasyliunas, 1983). It has recently been proposed that Jupiter's main auroral oval is formed in this way (Bunce and Cowley, 2001; Cowley and Bunce, 2001; Hill, 2001; Southwood and Kivelson, 2001), the angular momentum transfer being required by the equatorial radial outflow of sulphur and oxygen plasma from the Io plasma torus. At Saturn, the magnetospheric plasma source, originating from icy moons and ring grains, is believed to be on a lesser scale than at Jupiter by more than an order of magnitude (e.g. Richardson, 1992; Richardson et al., 1998). However, as shown directly by Voyager observations, it is still sufficient to cause significant sub-corotation of the equatorial magnetospheric plasma at radial distances beyond $\sim 5 R_{S}$ (Richardson 1986, 1995; Richardson and Sittler, 1990), such that the same coupling current system is required to flow. ( $R_{S}$ here is the conventional Saturn radius equal to $60330 \mathrm{~km}$, and we note for comparison that the equatorial distance of the near-subsolar magnetopause is $\sim 20 R_{S}$ (Behannon et al., 1983)). Recently, however, Cowley and Bunce (2003) have examined the properties of this current system at Saturn, using empirical models of the magnetic field and plasma flow based on Voyager data, together with estimates of the effective Pedersen conductivity of the kronian ionosphere obtained by Bunce et al. (2003) from Voyager field data. The results show that the ionospheric upward field-aligned current is both too weak and flows at too low a latitude $\left(\sim 20^{\circ}\right.$ co-latitude) to correspond to the auroral currents responsible for Saturn's auroras. In addition, the currents associated with plasma sub-corotation are not expected to vary strongly with local time (except possibly between noon and midnight due to the asymmetry imposed by the solar wind flow), nor with universal time, unlike the auroras observed. 


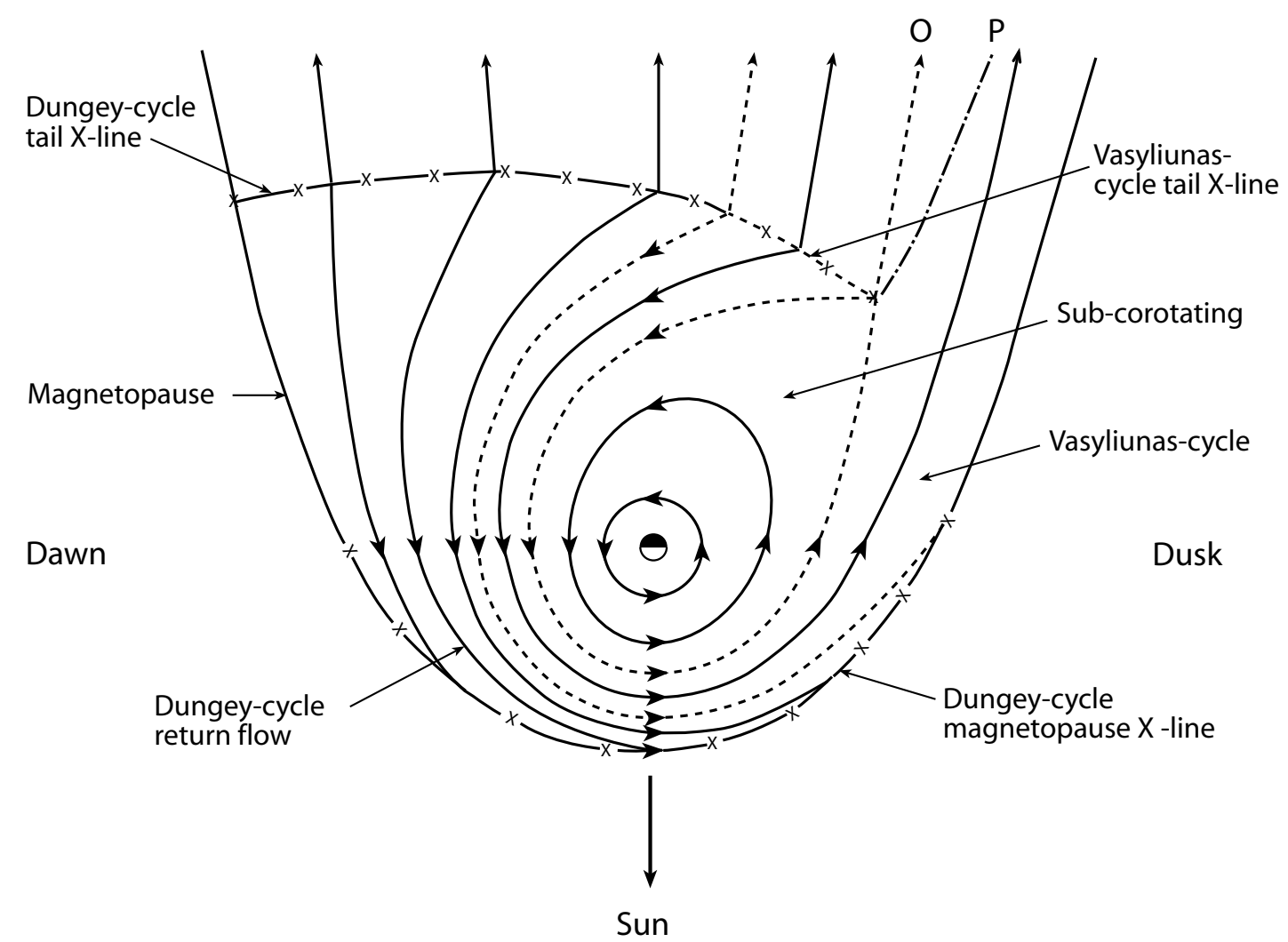

Fig. 2. Sketch of the plasma flow in the equatorial plane of Saturn's magnetosphere, where the direction to the Sun is at the bottom of the diagram, dusk is to the right, and dawn to the left. Arrowed solid lines show plasma streamlines, arrowed short-dashed lines the boundaries between flow regimes (also streamlines), the solid lines joined by Xs the reconnection lines associated with the Dungey cycle, and the dashed lines with Xs the tail reconnection line associated with the Vasyliunas cycle. The two tail reconnection lines are shown as being contiguous, but this is not necessarily the case. The line indicated by the "O" marks the path of the plasmoid O-line in the Vasyliunas-cycle flow (also a streamline), while "P" marks the outer limit of the plasmoid field lines, which eventually asymptotes to the dusk tail magnetopause.

The second possibility is that Saturn's auroras are instead associated with solar wind-magnetosphere coupling, as at Earth. In this case we would generally expect the auroras to lie in the vicinity of the boundary between open and closed field lines, which is located at co-latitudes of $\sim 11^{\circ}-15^{\circ}$, according to estimates based on Voyager-1 tail magnetic field data (Ness et al., 1981). This suggestion is thus reasonably consistent with the observed co-latitudes of the auroras, $\sim 10^{\circ}-18^{\circ}$ as indicated above. It is also supported by the finding that the apparently-related SKR emissions are positively correlated with the dynamic pressure of the solar wind (Desch, 1982; Desch and Rucker, 1983). In this paper we thus consider the polar flows and current systems at Saturn which are associated with the combined action of planetary rotation and solar wind-driven convection, and their relationship with the auroras.

\section{Equatorial flows}

The conceptual picture of the large-scale flow in Saturn's magnetosphere which forms the basis of our discussion derives from earlier suggestions by Cowley et al. (1996) for the jovian magnetosphere, based on spacecraft flyby observations. The consequences of this picture for the flows and currents in Jupiter's ionosphere have recently been discussed by Cowley et al. (2003b), and are applied here similarly to Saturn. Figure 2 shows our picture of the flows in the equatorial plane of Saturn's magnetosphere in a view in which the direction towards the Sun is at the bottom of the figure, dusk is to the right, and dawn to the left. The arrowed solid lines show plasma streamlines, while the arrowed dashed lines (also streamlines) indicate the boundaries of the various components of the flow. Three such components are depicted. In the innermost region the flow is dominated by corotation with the planet. The streamlines are thus closed around the planet, though extending to larger distances on the nightside than on the dayside in the outer part due to the dayside confining effect of the solar wind flow. The physics of this region is dominated by plasma production and pick-up from neutral gas sources originating from ring grains and the icy surfaces of moons, followed by centrifugally-driven outward radial transport associated with small-scale motions not illustrated in the figure (e.g. Johnson et al., 1989; Pospieszalska and Johnson, 1991; Richardson, 1992; Richardson et al., 1998). No detailed self-consistent dynamical models of these 
processes have yet been proposed for Saturn as they have for Jupiter (e.g. Hill, 1979, 2001; Pontius, 1997; Cowley et al., 2002; Nichols and Cowley, 2003). However, Voyager plasma observations in the dayside equatorial magnetosphere show that the plasma angular velocity falls from near-rigid corotation close to the planet, within $\sim 5 R_{S}$, to values of $\sim 50 \%$ of rigid corotation at distances of $\sim 15 R_{S}$, within a few $R_{S}$ of the dayside magnetopause (Richardson, 1986). Empirical models of the equatorial flow in Saturn's magnetosphere have been developed from this data (Richardson and Sittler, 1990; Richardson, 1995; Cowley and Bunce, 2003), and form a basis for the discussion given here.

The ultimate loss process of the outward-diffusing kronian plasma is at present unknown. However, following previous suggestions for Jupiter, initially by Vasyliunas (1983), we depict in Fig. 2 a surrounding outer region, delimited by the arrowed dashed lines, in which sub-corotating planetary plasma is lost by outflow down the dusk flank magnetotail. The outer mass-loaded field lines, which are confined on the dayside by the solar wind, are envisaged to stretch out downtail as they rotate into the dusk sector, followed by reconnection in the plasma sheet, plasmoid formation, and pinch-off of the distended tailward-flowing closed flux tubes. This is illustrated in Fig. 2 as a steady-state process in which the associated reconnection $\mathrm{X}$-line is shown by the dashed line with Xs, labelled "Vasyliunas-cycle tail X-line". In reality, the process is probably highly time-dependent, as recent evidence suggests it to be at Jupiter (Woch et al., 2002). The magnetic O-line of the plasmoid, which is also a streamline, is indicated by the arrowed dashed line marked "O", while the outer boundary of the plasmoid (not a streamline) is indicated by the dot-dashed line marked "P", which asymptotes to the dusk magnetopause at large distances. Thus we envisage that the central region of sub-corotational flow is surrounded in the post-midnight, dawn, and morning sectors by a region of somewhat enhanced flow speeds, as the mass-reduced flux tubes flow sunward downstream of the Vasyliunas-cycle tail reconnection site under the action of the ionospheric torque. We note that Voyager plasma data indeed indicate the presence of somewhat variable but typically elevated azimuthal flow speeds in the noon sector at equatorial distances beyond $\sim 15 R_{S}$, with values typically between $\sim 100-150 \mathrm{~km} \mathrm{~s}^{-1}$ (Richardson, 1986), corresponding to $\sim 60-80 \%$ of rigid corotation. However, the flow on these flux tubes will slow as they rotate further into the dusk sector, becoming loaded once more with outward-moving planetary plasma.

The third component of flow shown in Fig. 2 is then the outermost region driven by the magnetospheric interaction with the solar wind, which we suppose is dominated by reconnection and the Dungey cycle (Dungey, 1961). These flows are initiated by reconnection between the southwarddirected planetary field in the equatorial magnetosphere and a preferentially northward-pointing magnetosheath field deriving from the interplanetary magnetic field (IMF). In Fig. 2 the reconnection sites are supposed to be broadly distributed over the dayside magnetopause, as shown by the solid lines with Xs labelled "Dungey cycle magnetopause X-line". Flux tubes in the outer dawn magnetosphere flow sunward to the magnetopause where reconnection takes place, the resulting "open" field lines then being carried over the poles of the planet, and out of the plane of the diagram, by the magnetosheath flow. The open flux tubes then reside in the tail lobes for an interval (to be discussed further below), before reconnecting again in the tail centre plane at the "Dungey cycle tail X-line", also shown by the solid lines with Xs. Closed flux tubes then return sunward once more to complete the cycle, also contributing to the observed region of somewhat elevated azimuthal flows in the outer region near noon. It can be seen from Fig. 2 that we envisage the return flow to take place solely via the dawn meridian. This is due first to the ionospheric torque imposed on the reconnected closed flux tubes which sweeps them towards the dawn side of the magnetosphere, and second to the outflow of planetary plasma that occupies the dusk side. Thus we envisage the Dungeycycle tail X-line to be confined to the midnight and dawn sectors of the tail. This X-line is shown in Fig. 2 to be spatially contiguous with the Vasyliunas cycle X-line in the dusk sector, though this is not necessarily the case. One further consequence of this configuration is that the open flux tubes in the tail will generally flow across the lobes from dusk to dawn in moving from the magnetopause to the tail reconnection sites. The typical flux transport in the Dungey cycle component of the flow can be estimated as some fraction of the solar wind voltage across a magnetospheric diameter. Taking, for example, an interplanetary field strength of $\sim 0.5 \mathrm{nT}$ (about a tenth of that at Earth), a magnetospheric diameter of $\sim 80 R_{S}$ (Ness et al., 1981), and a solar wind speed of $\sim 500 \mathrm{~km} \mathrm{~s}^{-1}$, yields a total cross-magnetosphere voltage in the undisturbed solar wind of $\sim 1 \mathrm{MV}$. If we assume that the reconnection process is about $\sim 15 \%$ efficient, as at Earth (e.g. Reiff et al., 1981), then the flux transport in the Dungey cycle is estimated to be typically equivalent to a voltage of $\sim 200 \mathrm{kV}$ (though of course modulated about this value by the orientation of the IMF). With azimuthal flow speeds in the outer dayside magnetosphere of $\sim 100-150 \mathrm{~km} \mathrm{~s}^{-1}$, as indicated above, and field strengths adjacent to the magnetopause of $\sim 5-10 \mathrm{nT}$ (Smith et al., 1980; Ness et al., 1981, 1982), the implied total thickness of the dayside layer of Dungey cycle "return" flows is $\sim 4 R_{S}$, or $\sim 2 R_{S}$ in the vicinity of noon, in rough conformity with Fig. 2. (The voltage $\Phi$ across a layer of width $W$ transverse to a field of strength $B$ with a transverse plasma flow of speed $V$ is $\Phi=V B W$, from which $W$ can be computed if $\Phi, V$, and $B$ are known.) The flux transport in the Vasyliunas cycle in Fig. 2 has been assumed to be of comparable order. This is, of course, conjectural at the present time, but is at least consistent with the observed $\sim 5 R_{S}$ layer of somewhat elevated azimuthal flow speeds adjacent to the dayside magnetopause mentioned above. By comparison, the voltage across the corotating region from the planetary equator out to $\sim 15 R_{S}$ near noon is $\sim 10 \mathrm{MV}$. 


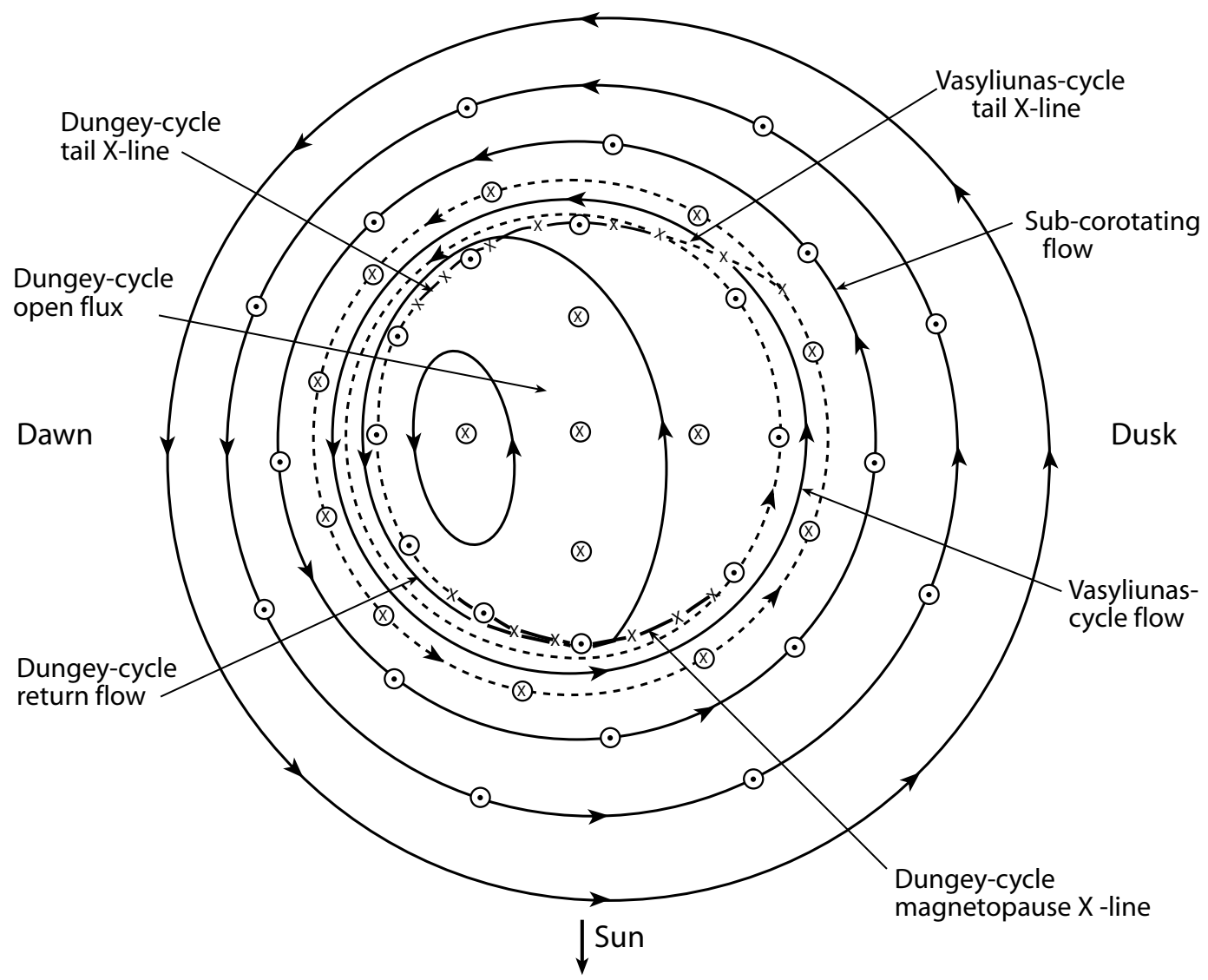

Fig. 3. Sketch of the plasma flow in the northern kronian ionosphere in a format following that of Fig. 2, where the direction to the Sun is at the bottom of the diagram, dusk is to the right, and dawn to the left. The outermost circle corresponds to a co-latitude of $\sim 30^{\circ}$ from the pole, which maps to the equatorial plane at a radial distance of $\sim 3 R_{S}$. Circled dots and crosses indicate regions of upward and downward fieldaligned current, respectively, as indicated by the divergence of the horizontal ionospheric current. Hall currents flow generally anti-clockwise round the pole and close in the ionosphere, while Pedersen currents flow generally equatorward and close in the field- aligned current system shown.

\section{Ionospheric flows and currents}

In this section we now discuss the flows in Saturn's ionosphere that correspond to those in the equatorial magnetosphere just outlined. These are depicted in Fig. 3, where we use the same line formats as in Fig. 2. One feature shown in Fig. 3 which could not be shown in Fig. 2, however, is the "polar cap" region of open flux, which maps to the tail lobes and thus lies outside of the equatorial plane. This is located in the inner region of the figure. It is bounded in the noon and midnight sectors by the dayside and nightside "merging gaps", where the field lines map to the magnetopause and tail Dungey-cycle reconnection X-lines. Plasma and frozenin flux tubes flow across the boundary from the closed to the open region at the dayside merging gap, mapping to the magnetopause reconnection sites, while flowing from the open to the closed region at the nightside merging gap mapping to the tail reconnection sites. Between the merging gaps, "adiaroic" portions of the boundary span dawn and dusk (dashed lines), mapping to the flank magnetopauses. The adiaroic portions of the open-closed field line boundary are those not mapping to active reconnection sites, such that the normal component of the plasma velocity at the boundary is zero in the boundary rest frame (i.e. the plasma does not move across these portions of the boundary). As indicated in the Introduction, the size of the polar cap can first be estimated from the magnetic flux content of the tail lobes, obtained from flyby magnetic field data. Assuming a circular polar cap centred on the pole, Ness et al. (1981) estimated the boundary to be located at co-latitudes $\sim 11^{\circ}-15^{\circ}$ from Voyager- 1 data obtained at down-tail distances of $\sim 25 R_{S}$ (the furthest distance downtail explored by any of the three flyby spacecraft to date). Estimates can also be made by examining the flux content in the equatorial magnetosphere out to the magnetopause, using magnetic models derived from flyby data (Connerney et al., 1983; Bunce and Cowley, 2003). Values obtained on this basis yield boundary values of $\sim 12^{\circ}-13^{\circ}$ in the Northern Hemisphere and $\sim 13^{\circ}-15^{\circ}$ in the Southern Hemisphere (Cowley and Bunce, 2003), the difference being due to the effect of the quadrupole component of the internal field. We neglect this difference in the outline discussion given here, and will simply take the boundary to lie at $\sim 15^{\circ}$ co-latitude, 
as a round value. This corresponds to a perpendicular distance from the axis of the flattened planet of $\sim 14400 \mathrm{~km}$. We note for future reference that with a planetary angular velocity of $1.64 \times 10^{-4} \mathrm{rad} \mathrm{s}^{-1}$, the rigid corotation velocity at the boundary is then $\sim 2.4 \mathrm{~km} \mathrm{~s}^{-1}$.

In Fig. 3, however, we also show the centre of the open flux region to be displaced somewhat away from Saturn's pole, towards the nightside and towards dawn (effects we have also not incorporated into the simple calculations that follow). The displacement towards the nightside is simply due to the expected day-night asymmetry imposed on the outer magnetosphere by the solar wind flow. The displacement towards dawn, at least on the nightside, follows our related suggestion for Jupiter (Cowley et al., 2003b), and is a consequence of the outflow of planetary plasma down the dusk-side tail in the Vasyliunas cycle. This outflow displaces the Dungey-cycle open field lines across the tail lobe from dusk to dawn towards the reconnection sites in the midnight and dawn sector, thus also producing a dawnward component of flow across the polar cap towards a nightside merging gap which is similarly displaced in the dawnward direction. We envisage a dayside merging gap, mapping to the magnetopause reconnection sites, which is positioned roughly symmetrically with respect to noon, unless displaced, for example, by asymmetry effects associated with the prevailing IMF direction (see Cowley et al. (1991) for a discussion of the terrestrial case).

Given the size of the open field line region drawn in Fig. 3, the outer bounding streamline in the figure then corresponds to a co-latitude of $\sim 30^{\circ}$. The field lines from this outer region then map into the inner magnetosphere at radial distances of $\sim 3 R_{S}$ in the equatorial plane, where the plasma near-rigidly corotates with the planet (Richardson, 1986; Richardson and Sittler, 1990). On moving towards the pole, however, and hence radially outward in the equatorial magnetosphere, the plasma angular velocity starts to fall below that of rigid corotation, as discussed above. This decline is accompanied by a growth in the ionospheric Pedersen current, directed equatorward, and a related growth in the ionospheric Hall current, directed eastward, as may be deduced from the following considerations. It should be recalled that the ionospheric currents are determined by the strength and direction of the ionospheric electric field in the rest frame of the neutral atmosphere. The atmosphere rotates eastward with the planet at an angular velocity which is higher than that of the plasma, though it may be reduced somewhat below that of rigid corotation in the Pedersen layer due to ion-neutral frictional drag (see, Huang and Hill (1989) for a discussion in the jovian context). Consequently, in the rest frame of the neutral atmosphere, subcorotating plasma flows westward, such that the ionospheric electric field is directed equatorward. Thus, sub-corotation of the plasma results in equatorward-directed Pedersen currents and eastward-directed Hall currents being driven in the ionosphere, as just indicated, whose magnitudes increase as the departure from rigid corotation increases.
Both these current components thus grow with increasing latitude as the plasma angular velocity falls from rigid corotation. However, whereas the ionospheric Hall currents flow approximately around constant latitude paths and thus close within the ionosphere, the latitude gradient of the equatorward Pedersen current implies the existence of upwarddirected field-aligned currents in this region. Taking the Northern Hemisphere for definiteness, and assuming axial symmetry and a spherical planet of radius $R_{S}$ with a vertical polar magnetic field as sufficient first approximations, the field-aligned current density just above the current-carrying layer of the ionosphere is given by

$j_{\|}=-\frac{1}{2 \pi \rho R_{S}} \frac{d I_{P}}{d \theta}$,

where positive values indicate radially outward currents parallel to the field, $\rho=R_{S} \sin \theta$ is the perpendicular distance from the magnetic (and spin) axis at co-latitude $\theta$, and $I_{P}$ is the total equatorward-directed height-integrated Pedersen current flowing at that co-latitude, integrated in azimuth around the pole. Further assuming that the polar magnetic field is of approximately constant strength $B_{P S}$, the total Pedersen current $I_{P}$ is given by

$I_{P}=2 \pi \Sigma_{P}^{*} \Omega_{S} B_{P S} \rho^{2}\left(1-\frac{\omega}{\Omega_{S}}\right)$,

where $\Sigma_{P}^{*}$ is the "effective" height-integrated ionospheric Pedersen conductivity, possibly reduced from the true value by "slippage" of the neutral atmosphere due to ion-neutral frictional drag, as mentioned above, $\Omega_{S}$ is Saturn's angular velocity, and $\omega$ is the angular velocity of the plasma on the flux shell that maps to co-latitude $\theta$. As the plasma increasingly departs from rigid corotation with increasing latitude, therefore, so the total equatorward Pedersen current grows in accordance with Eq. (1b), thus requiring the presence of an upward-directed field-aligned current given by Eq. (1a), as indicated in Fig. 3 by the outer region of circled dots.

It will be noted in Eq. (1b), however, that the total Pedersen current is also proportional to the square of the distance from the magnetic axis. One factor of $\rho$ arises from the integration in azimuth, while the other arises from the fact that for a given plasma angular velocity $\omega$, the actual velocity of the plasma, and hence the electric field and current, are also proportional to $\rho$. Thus, even for a plasma angular velocity profile which declines monotonically with increasing latitude, as employed in the baseline models of Cowley and Bunce (2003) mentioned in the Introduction, the total current $I_{P}$ will eventually peak and then fall with increasing latitude, thus leading to a reversal in the sense of the field-aligned current at higher latitudes, given by Eq. (1a). In the Cowley and Bunce (2003) models the upward-directed field-aligned current was found to peak at a value of $5-10 \mathrm{nA} \mathrm{m}^{-2}$ at a colatitude of $\sim 20^{\circ}$, mapping to the equatorial plane at $\sim 7 R_{S}$, before declining and then reversing in sense to a downwarddirected current poleward of $\sim 16^{\circ}$, mapping equatorially to the region beyond $\sim 13 R_{S}$. In the augmented picture presented here, however, the reversal to downward field-aligned 
current should be accentuated by the enhanced angular velocities, and hence reduced Pedersen current intensities, that are expected to occur in the circumpolar band of flow mapping to the Vasyliunas-cycle, as well as in the dawn-sector Dungeycycle return flows, as mentioned above. These downward currents are indicated by the ring of circled crosses located equatorward of the open-closed field line boundary in Fig. 3. The upward and downward currents in this closed field line regime then close in the equatorial magnetospheric plasma in field-perpendicular currents which flow radially outward from the planet. The $\boldsymbol{j} \times \boldsymbol{B}$ force of the closure current imposes a torque on the equatorial plasma in the sense of planetary rotation, while the equal and opposite torque on the ionospheric plasma associated with the Pedersen current balances the torque due to ion-neutral collisions. The effect overall is to transfer angular momentum from the neutral atmosphere to the equatorial plasma.

We now consider the flows associated with the Dungeycycle in more detail. We first note that, in conformity with the above discussion, the Dungey cycle "return" flows from the tail take place only via dawn, due to the planetary torque on the newly-closed flux tubes, together with the outflow of planetary plasma in the dusk plasma sheet. The Dungey cycle thus gives rise only to a single cell of flow in the polar ionosphere in this case, rather than to the twin-cell flow which is generated at Earth. We also note that the "return" flow should occupy only a narrow latitudinal channel in the dawn ionosphere, as shown in Fig. 3. If we assume, for example, that the flow in this region corresponds to, say, $\sim 80 \%$ of rigid corotation, with a consequent flow speed in the ionosphere of $\sim 1.9 \mathrm{~km} \mathrm{~s}^{-1}$ (rigid corotation gives $\sim 2.4 \mathrm{~km} \mathrm{~s}^{-1}$ at the boundary as noted above), then, if the total voltage across the layer is $\sim 200 \mathrm{kV}$, as also indicated above, the latitudinal width of the layer implied is $\sim 1500 \mathrm{~km}$, or a little less than $1.5^{\circ}$ of latitude. Since the region of open field lines is substantially wider than this, by roughly an order of magnitude, as indicated by the estimates given above, the implication is that the anti-sunward Dungey-cycle flow within the region of open field lines is much slower than the "return" flows, also by roughly an order of magnitude. A specific estimate of the polar cap flow speed can be made by considering the time taken by an open flux tube to flow through the tail lobe from the magnetopause to the tail centre plane, this being equal to the time it takes in the ionosphere for the same open flux tube to move from the dayside to the nightside merging gaps. For a voltage of $\sim 200 \mathrm{kV}$ across a tail of diameter $\sim 80 R_{S}$ with a lobe field strength of $\sim 3 \mathrm{nT}$, as observed by Voyager- 1 (Ness et al., 1981), the flow speed in the tail lobe is $\sim 15 \mathrm{~km} \mathrm{~s}^{-1}$, and the total travel time is $\sim 50 \mathrm{~h}$, corresponding to $\sim 5$ planetary rotations. The corresponding transpolar flow speed in the polar ionosphere is $\sim 200 \mathrm{~m} \mathrm{~s}^{-1}$, a tenth of that of the return flows, as indicated above. By comparison, the travel time in the "return" flow from the tail to the dayside implied by the above numbers is $\sim 5-10 \mathrm{~h}$.

In addition to the Dungey-cycle flow within the polar region, however, there is another component of flow on the open field lines which has not yet been considered here, this being due to the atmospheric frictional torque associated with ion-neutral collisions in the Pedersen layer, which tends to rotate the flux tubes in the polar ionosphere, and hence, to twist the open field lines in the tail lobes. This problem has been considered by Isbell et al. (1984), who, given some assumptions of simplified geometry, showed that in the steady state, the ionospheric plasma sub-corotates with angular velocity

$\omega=\frac{\mu_{0} \Sigma_{P}^{*} V_{S W}}{1+\mu_{0} \Sigma_{P}^{*} V_{S W}} \Omega_{S}$,

where $V_{S W}$ is the speed of the solar wind. Now, while the value of $V_{S W}$ at Saturn's orbit is well-constrained, typically to within a factor of two, the same cannot be said for the value of the effective height-integrated Pedersen conductivity $\Sigma_{P}^{*}$. A number of estimates have been made of the true value of the Pedersen conductivity based on observed and modelled electron density profiles, giving values varying between $\sim 0.1$ and $\sim 100$ mho (e.g. Connerney et al., 1983; Atreya et al., 1984; Cheng and Waite, 1988). Isbell et al. (1984) themselves adopted a conductivity of 10 mho, yielding a value for the dimensionless quantity $\mu_{0} \Sigma_{P}^{*} V_{S W} \approx 6.3$ for a solar wind speed of $\sim 500 \mathrm{~km} \mathrm{~s}^{-1}$. Since this value is significantly larger than unity, the angular velocity given by Eq. (2) is close to rigid corotation with the planet. Specifically for the above numbers we find $\omega \approx 0.86 \Omega_{S}$. In this case the tail field lines are twisted at near the maximal (infinite conductivity) rate $\omega=\Omega_{S}$. However, Bunce et al. (2003) have recently made an empirical estimate of $\Sigma_{P}^{*}$ for the closed field region of Saturn's ionosphere, by examining the "bending back" of magnetospheric field lines observed during the Voyager flybys which is associated with the magnetosphere-ionosphere coupling current circuit described for the closed field line region above. Their results place an upper limit on the effective Pedersen conductivity of the conjugate ionosphere of $\sim 1-2$ mho. Given that this is an upper limit, and that hot plasma precipitation from the tail lobe should be minimal in the polar ionosphere (as we will confirm below), the effective conductivity of the polar ionosphere is likely to be less than this, and hence, considerably less than the value taken by Isbell et al. (1984). If we, instead, take $\Sigma_{P}^{*} \approx 0.5 \mathrm{mho}$, for example, we then find $\mu_{0} \Sigma_{P}^{*} V_{S W} \approx 0.31$, i.e. a value rather less than unity. In this case the ionospheric plasma only weakly rotates due to the atmospheric torque, at an angular velocity given by Eq. (2) for the above numbers of $\omega=\approx 0.24 \Omega_{S}$. We regard this as being the more likely scenario at Saturn, compared with the near-rigid corotation deduced by Isbell et al. (1984) in this case. (We also note in passing that the likely value of $\Sigma_{P}^{*}$ for Jupiter's polar ionosphere is of the order of $\sim 0.1 \mathrm{mho}$, such that $\mu_{0} \Sigma_{P}^{*} V_{S W} \approx 0.06$ and $\omega \approx 0.05 \Omega_{J}$, justifying the neglect of this effect at Jupiter by Cowley et al. (2003b).)

Assuming the validity of the above estimates, we then find that the speed of the rotating ionospheric flow within Saturn's open field region is modest, and comparable in magnitude with the transpolar speed associated with the Dungey cycle estimated above. For example, the maximum speed at the 
boundary of the region of open flux at a co-latitude of $\sim 15^{\circ}$ is $\sim 550 \mathrm{~m} \mathrm{~s}^{-1}$, compared with $\sim 200 \mathrm{~m} \mathrm{~s}^{-1}$ for the Dungey cycle. Keeping in mind the direction of these flows, we see that the combined flow will be anti-sunward at $\sim 750 \mathrm{~m} \mathrm{~s}^{-1}$ at the dusk edge of the polar cap, while being sunward at $\sim 350 \mathrm{~m} \mathrm{~s}^{-1}$ at the dawn edge. The results of these estimates are incorporated qualitatively into the sketch of the polar flow streamlines shown in Fig. 3, where we show, for example, a closed flow vortex in the dawn sector of the polar cap. We do not wish to imply by this that regions exist within the polar cap and tail lobe where open field lines circulate indefinitely, but closed streamlines can certainly exist for an interval between bursts of more vigorous Dungey-cycle activity driven either from the magnetopause or from the tail.

We also note in passing that these results provide information on the length of Saturn's magnetic tail. In general, the length of the open tail field lines can be estimated by computing the distance travelled by the solar wind during the time for which a typical open field line remains open (Dungey, 1965). For a tail dominated by Dungey-cycle flow, this time is the time taken by an open flux tube to drift from the magnetopause to the tail centre under the action of the cross-tail electric field, this being typically $\sim 50 \mathrm{~h}$ for Saturn, as estimated above. However, in a twisting tail, portions of the open field lines can also be convected into the tail centre by rotation from the ionosphere about the tail axis, following which reconnection and closure can also occur. The time scale for this is roughly one period of rotation, which is also $\sim 50 \mathrm{~h}$ for $\omega \approx 0.24 \Omega_{S}$. In general, the time scale which is germane is the shorter of the two, but in this case we estimate comparable time scales of $\sim 50 \mathrm{~h}$. The corresponding length of Saturn's magnetic tail is thus estimated to be $\sim 1500 R_{S}$ $\left(\sim 9 \times 10^{7} \mathrm{~km}\right)$.

The central point we wish to emphasise from this discussion, however, is that the flow within the region of open field lines is expected typically to be slow, of the order of several $100 \mathrm{~m} \mathrm{~s}^{-1}$, but surrounded by much faster eastward flows on closed field lines, with speeds of $\sim 1-2 \mathrm{~km} \mathrm{~s}^{-1}$, as depicted in Fig. 3. The consequences for the ionospheric field-aligned current flow is also shown in the figure. First, because the plasma flow speed within the region of open field lines is small compared with that of the neutral atmosphere, with plasma angular velocities typically $\sim 0.1-0.3 \Omega_{S}$, compared with a neutral atmosphere angular velocity of $\sim \Omega_{S}$, an equatorward-directed Pedersen current will flow throughout the polar cap ionosphere, with a strength which is approximately proportional to distance from the planetary rotation axis (see Eq. 1b). This current implies the existence of a downward-directed field-aligned current throughout this region which is approximately spatially uniform, as indicated in Fig. 3. The downward current is consistent with the sense of twisting of the open tail field lines as discussed above, and as previously discussed by Isbell et al. (1984), and is also consistent with a lack of accelerated particle precipitation into this region, as indicated above. However, at the boundary between the open and closed field lines the Pedersen current is suddenly reduced by the increase in plasma flow speed on closed field lines to angular velocities which may be only a modest factor smaller than that of the neutral atmosphere, i.e. to plasma angular velocities of, say $\omega \approx 0.6-0.8 \Omega_{S}$, as indicated in the previous section. Current continuity then requires a ring of upward-directed field-aligned current to flow at the boundary between open and closed field lines, as also shown in Fig. 3. This current closes in the downward currents within the region of open field lines via field-perpendicular currents flowing in the magnetopause and boundary layers. Here we suggest that it is this ring of upward field-aligned current that is associated with the main oval auroras observed at Saturn. The existence of such a current is in accord with the previous discussion given by Isbell et al. (1984). However, it should be emphasised that the actual sense of the field-aligned current at the boundary depends on the change in plasma angular velocity (and possibly ionospheric conductivity) that occurs there. Under the conditions which Isbell et al. suggested, in which the plasma on open field lines near-rigidly corotates with the planet due to high assumed ionospheric conductivity, the field-aligned current flow at the boundary would actually be reversed compared with that envisaged here, if the closed field lines rotate with a lesser angular velocity, comparable to those actually observed in the outer magnetosphere.

While the upward-directed field-aligned current marking the open-closed field line boundary is expected to form a continuous ring on the basis of the above discussion, the currents should be stronger at dawn than at dusk, due to the greater shear in the flow at the dawn boundary than at dusk. This will also lead to a similar asymmetry in the brightness of related auroras as discussed in the following section. The dawn-dusk asymmetry in the flow shear results from two related effects. We have already pointed out above that the combined effect of atmosphere-driven plasma rotation and Dungey-cycle flow in the open field line polar cap will typically result in corotation-directed flows at the boundary of the region of $\sim 350 \mathrm{~m} \mathrm{~s}^{-1}$ at dawn and $\sim 750 \mathrm{~m} \mathrm{~s}^{-1}$ at dusk, thus being smaller at dawn than at dusk due to the Dungeycycle flow. On the other hand, the speed of closed field lines on the equatorward side of the boundary should be greater at dawn than at dusk, since the closed field lines at the dawn boundary correspond to the return flows of the Dungey cycle downstream from the tail reconnection site, involving flux tubes which should not be significantly loaded with planetary plasma, while those on the dusk side correspond to the more mass-loaded flux tubes of the Vasyliunas cycle as they stretch out down the tail before pinching off (see Fig. 3). For sake of argument, then, let us suppose that the angular velocity of the closed flux tubes at dawn corresponds to $\sim 80 \%$ of rigid corotation, as already employed above, while that of the closed flux tubes at dusk corresponds to, say, $\sim 60 \%$ of rigid corotation, with these values at least being plausible in terms of the Voyager plasma velocity observations in the outer magnetosphere mentioned previously. Then the flow speed of the closed field lines on the equatorward side of the boundary will be $\sim 1900 \mathrm{~m} \mathrm{~s}^{-1}$ at dawn and $\sim 1400 \mathrm{~m} \mathrm{~s}^{-1}$ at dusk. Consequently, with these numbers, the flow shear 
will be $\sim 1550 \mathrm{~m} \mathrm{~s}^{-1}$ at dawn compared with $\sim 650 \mathrm{~m} \mathrm{~s}^{-1}$ at dusk. If we then take a value of the effective Pedersen conductivity of the ionosphere of $\sim 0.5 \mathrm{mho}$, as employed above (for simplicity assumed to be the same on both sides of the boundary), and a polar field strength of $\sim 65000 \mathrm{nT}$, the implied intensity of the equatorward-directed horizontal Pedersen current at the boundary in the dawn sector is $\sim 65 \mathrm{~mA} \mathrm{~m}^{-1}$ on open field lines, and $\sim 15 \mathrm{~mA} \mathrm{~m}^{-1}$ on adjacent closed field lines, resulting in an upward-directed fieldaligned current, integrated across the width of the boundary, of $\sim 50 \mathrm{~mA} \mathrm{~m}^{-1}$. By comparison, in the dusk sector the Pedersen currents are estimated as $\sim 50 \mathrm{~mA} \mathrm{~m}^{-1}$ on open field lines and $\sim 30 \mathrm{~mA} \mathrm{~m}^{-1}$ on adjacent closed field lines, resulting in a reduced upward current intensity of $\sim 20 \mathrm{~mA} \mathrm{~m}^{-1}$. At other local times the upward current should take intermediate values between these extremes, with an averaged value of $\sim 35 \mathrm{~mA} \mathrm{~m}^{-1}$. Thus, we estimate the total upwarddirected field-aligned current integrated around the ring to be $\sim 3 \mathrm{MA}$, i.e. a total of $\sim 5 \mathrm{MA}$ flows into the boundary region from the polar cap ionosphere, $\sim 2 \mathrm{MA}$ similarly flows out into the closed field ionosphere, and $\sim 3$ MA returns up the field lines in the boundary. The peak ionospheric current flowing in the corotation-enforcement circuit at lower latitudes was estimated by Cowley and Bunce (2003) to be $\sim 6 \mathrm{MA}$ (assuming a slightly elevated effective Pedersen conductivity on inner closed field lines of $1 \mathrm{mho}$ ). If correct, this implies that 4 MA of distributed downward current then flows between the boundary of open-closed field lines and the position of the peak ionospheric Pedersen current at $\sim 20^{\circ}$. The full $\sim 6 \mathrm{MA}$ then flows out of the ionosphere in the equatorward region, as the plasma reaches rigid corotation with the planet. If, instead, we assume a constant effective ionospheric conductivity of 0.5 mho throughout, then the peak Pedersen current at lower latitudes reduces to $\sim 3 \mathrm{MA}$, implying that the additional downward current flowing on closed field lines is $\sim 1 \mathrm{MA}$, with $\sim 3 \mathrm{MA}$ then flowing up and out of the ionosphere at latitudes below the peak. In either case, the upward current is comparable with, or larger than, the estimate above for the total current flowing out of the ionosphere in the boundary between open and closed field lines. However, the corotation-related upward-directed fieldaligned currents are expected to be distributed over $\sim 10^{\circ}$ of latitude, rather than flowing in a narrow latitudinal layer like those near the boundary of open and closed field lines. The current densities are thus likely to be significantly higher in the latter case than in the former.

We finally note that the above estimates of the fieldaligned current intensities, integrated across the width of the open-closed field line boundary, do not depend on the details of the structure of the transition across the boundary, but only on the flows and conductivities in the unperturbed ionospheres on either side. In particular, the values are not effected by the possible presence of localised enhancements in the effective Pedersen conductivity of the ionosphere, produced by precipitating accelerated auroral electrons which carry the upward current in the layer, as will be discussed in more detail in the following section. However, such precip- itation will, of course, effect the structure and width of the current layer, in particular by elevating the conductivity and current in the high-flow low-current region on the equatorward side of the boundary. The field-aligned current layer may then extend across the open-closed field line boundary, preferentially into the region of closed field lines equatorward of the boundary, though detailed modelling of this effect is clearly beyond the scope of the present work.

\section{Auroral parameters}

In order to estimate the auroral accelerations required by the polar currents, we need to know not just the intensity of the field-aligned current flowing in the boundary (the current per unit length of boundary) as estimated above, but rather the value of the density of the field-aligned current in the boundary region. To obtain this quantity, we then need to know the width of the current layer, as well as the current intensity, a parameter that is not simply determined by the theoretical description given above, as just mentioned. However, empirical estimates can be made from the width of the observed auroras, such as those shown in Fig. 1. These observations indicate that the latitudinal widths of the precipitation regions, and hence of the upward field-aligned currents, lie in the range from $\sim 300$ to more than $\sim 1000 \mathrm{~km}(\sim 1000 \mathrm{~km}$ corresponds to $\sim 1^{\circ}$ of latitude), as indicated by the data given in Table 1. Here we will thus take three illustrative values spanning the likely range, which differ from each other by factors of two, i.e. 250,500 , and $1000 \mathrm{~km}$. For these values, the current densities will be $\sim 200,100$, and $50 \mathrm{nA} \mathrm{m}^{-2}$ at dawn, and $\sim 80,40$, and $20 \mathrm{nA} \mathrm{m}^{-2}$ at dusk. The peak values estimated by Cowley and Bunce (2003), associated with the large-scale corotation enforcement current system on closed field lines, are $\sim 5-10 \mathrm{nA} \mathrm{m}^{-2}$, smaller than any of these estimates. These values must be compared with the current densities that can be carried by magnetospheric electrons without field-aligned acceleration. This is given by

$j_{\| i 0}=e N\left(\frac{W_{t h}}{2 \pi m_{e}}\right)^{1 / 2}$,

where $N$ is the number density of the electrons (assumed isotropic), $W_{t h}$ is their thermal energy, and $m_{e}$ and $e$ are the electron mass and charge. Equation 3 corresponds to the condition of a full downward-going loss-cone in the magnetospheric electron population, and an empty upward-going loss-cone. The corresponding precipitated electron energy flux is

$E_{f 0}=2 N W_{t h}\left(\frac{W_{t h}}{2 \pi m_{e}}\right)^{1 / 2}$.

The auroral luminosity associated with a given electron energy flux can be estimated from the following considerations. Collisions of the electrons with atmospheric particles result in the emission of UV photons in the spectral range $\sim 85-175 \mathrm{~nm}$ (i.e. photons of energy $\sim 10 \mathrm{eV}$ ), corresponding to the $\mathrm{H}_{2}$ Lyman and Werner bands and the 
Table 2. Minimum field-aligned voltage $\Phi_{\min }$ and precipitating electron energy flux $E_{f}$ given by Eqs. (5) and (6) for (a) magnetosheath $\left(N=0.2 \mathrm{~cm}^{-3}\right.$ and $\left.W_{t h}=50 \mathrm{eV}\right)$ and (b) outer magnetospheric $\left(N=0.01 \mathrm{~cm}^{-3}\right.$ and $\left.W_{t h}=1 \mathrm{keV}\right)$ source electron populations, for both dawn and dusk conditions at the open-closed field line boundary (i.e. upward field-aligned current intensities of 50 and $20 \mathrm{~mA} \mathrm{~m}^{-1}$ respectively). Results are shown for three assumed widths of the current layer, 250, 500, and $1000 \mathrm{~km}$. Estimates of the UV auroral brightness (in kR) can be obtained by multiplying the precipitating electron energy flux (in $\mathrm{mW} \mathrm{m}^{-2}$ ) by a factor of ten.

(a) Magnetosheath electron source

\begin{tabular}{ccccccc}
\hline \multicolumn{7}{c}{ Width of field-aligned current layer } \\
\hline & $\Phi_{\min }(\mathrm{kV})$ & $E_{f}\left(\mathrm{~mW} \mathrm{~m}^{-2}\right)$ & $\Phi_{\min }(\mathrm{kV})$ & $E_{f}\left(\mathrm{~mW} \mathrm{~m}^{-2}\right)$ & $\Phi_{\min }(\mathrm{kV})$ & $E_{f}\left(\mathrm{~mW} \mathrm{~m}^{-2}\right)$ \\
\hline Dawn & 0.21 & 0.055 & 0.082 & 0.015 & 0.016 & 0.005 \\
\hline Dusk & 0.056 & 0.010 & 0.003 & 0.004 & 0.0 & 0.004 \\
\hline
\end{tabular}

(b) Magnetospheric electron source

\begin{tabular}{|c|c|c|c|c|c|c|}
\hline \multicolumn{7}{|c|}{ Width of field-aligned current layer } \\
\hline & \multicolumn{2}{|c|}{$250 \mathrm{~km}$} & \multicolumn{2}{|c|}{$500 \mathrm{~km}$} & \multicolumn{2}{|c|}{$1000 \mathrm{~km}$} \\
\hline & $\Phi_{\min }(\mathrm{kV})$ & $E_{f}\left(\mathrm{~mW} \mathrm{~m}^{-2}\right)$ & $\Phi_{\min }(\mathrm{kV})$ & $E_{f}\left(\mathrm{~mW} \mathrm{~m}^{-2}\right)$ & $\Phi_{\min }(\mathrm{kV})$ & $E_{f}\left(\mathrm{~mW} \mathrm{~m}^{-2}\right)$ \\
\hline Dawn & 22.6 & 4.7 & 10.8 & 1.2 & 4.9 & 0.30 \\
\hline Dusk & 8.4 & 0.76 & 4.7 & 0.20 & 1.4 & 0.056 \\
\hline
\end{tabular}

H Lyman lines. Taking an efficiency of the energy transfer of $\sim 14-18 \%$ (Waite et al., 1983; Rego et al., 1994), then yields a source brightness of $\sim 9-12 \mathrm{kR}$ for an electron energy flux of $1 \mathrm{~mW} \mathrm{~m}^{-2}$. Some of these photons are absorbed before they leave the atmosphere, however, depending on the altitude of emission. Examination of the auroral spectrum obtained a few minutes after the image shown in Fig. 1 indicates that the absorption is small, $\sim 10-20 \%$, such that we estimate that a precipitating electron energy flux of $1 \mathrm{~mW} \mathrm{~m}^{-2}$ produces an auroral brightness in the range $\sim 8-11 \mathrm{kR}$. These are the values employed to determine the energy flux ranges corresponding to the brightness ranges given in Table 1. For simple conversions in the theoretical discussion below, however, we will simply take $1 \mathrm{~mW} \mathrm{~m}^{-2}$ to correspond to $\sim 10 \mathrm{kR}$.

The field-aligned current considered here flows explicitly in a layer at the boundary between open and closed field lines. It may then be carried by cool magnetosheath (cusp) plasma in the poleward regions, and by hot magnetospheric electrons in the equatorward regions, though in the last section, we gave brief reasons to suppose that the current may extend principally into the region of closed field lines. However, for completeness we also consider the possibility of a cusp magnetosheath plasma source, and consider this case first. We may then estimate $N \approx 0.2 \mathrm{~cm}^{-3}$ (some fraction of the dayside magnetosheath density) and $W_{t h} \approx 50 \mathrm{eV}$ (for plasma heated at the magnetopause) (e.g. Sittler et al., 1983). We then find that the limiting current given by Eq. (3) is $\sim 40 \mathrm{nA} \mathrm{m}^{-2}$, while the limiting energy flux given by Eq. (4) is $\sim 0.004 \mathrm{~mW} \mathrm{~m}^{-2}$ (corresponding to an aurora of less than a few tens of R). Thus we find that field-aligned acceleration of electrons by parallel electric fields is required to produce the field-aligned currents at the dawn open-closed field line boundary under all conditions considered above, while acceleration is required at dusk only for the narrower current layers. To estimate the acceleration requirements, we use the kinetic theory presented by Knight (1973) (see also the discussion in Cowley and Bunce, 2001). This shows that the minimum field-aligned voltage required is

$\Phi_{\min }=\frac{W_{t h}}{e}\left[\left(\frac{j_{\| i}}{j_{\| i 0}}\right)-1\right]$,

giving a corresponding enhanced precipitating electron energy flux of

$E_{f}=\frac{E_{f 0}}{2}\left[\left(\frac{j_{\| i}}{j_{\| i 0}}\right)^{2}+1\right]$,

the latter result following Lundin and Sandahl (1978). Results are given in Table 2a, where for simplicity of initial estimate we have assumed a uniform electron source population across the whole current layer. These show that at dawn the required accelerating voltage increases from $\sim 20$ to $\sim 200 \mathrm{~V}$ as the layer width decreases from 1000 to $250 \mathrm{~km}$, with corresponding precipitating energy fluxes increasing from $\sim 0.005$ to $\sim 0.05 \mathrm{~mW} \mathrm{~m}^{-2}$. However, even the highest of these values corresponds to a UV emission of only $\sim 0.5 \mathrm{kR}$, so that the auroral luminosity is extremely feeble in this case. At dusk the values are even lower, with 
the minimum accelerating voltage increasing from zero to $\sim 50 \mathrm{~V}$ as the layer width decreases from 1000 to $250 \mathrm{~km}$, with precipitating energy fluxes increasing from the "unaccelerated" value of $\sim 0.004 \mathrm{~mW} \mathrm{~m}^{-2}$ to $\sim 0.01 \mathrm{~mW} \mathrm{~m}^{-2}$ ( $\sim 0.1 \mathrm{kR}$ or less). If, however, the current is instead carried by hot magnetospheric electrons, we may then take roughly $N \approx 0.01 \mathrm{~cm}^{-3}$ and $W_{t h} \approx 1 \mathrm{keV}$, values which are specifically appropriate to the hot electron component in the outer layers of the magnetosphere (e.g. Richardson and Sittler, 1990; Richardson, 1995). These numbers give a limiting current from Eq. (3) of $\sim 10 \mathrm{nA} \mathrm{m}^{-2}$, and a limiting energy flux from Eq. (4) of $\sim 0.02 \mathrm{~mW} \mathrm{~m}^{-2}$, corresponding to an "unaccelerated" UV aurora of $\sim 0.2 \mathrm{kR}$. In this case, therefore, auroral acceleration is required at all local times from dawn to dusk, for all widths of the current layer considered here. Results are shown in Table $2 \mathrm{~b}$. At dawn the accelerating voltage now increases from $\sim 5$ to $\sim 25 \mathrm{kV}$, as the layer width decreases from 1000 to $250 \mathrm{~km}$, with precipitating energy fluxes increasing from $\sim 0.5$ to $\sim 5 \mathrm{~mW} \mathrm{~m}^{-2}$, corresponding to $\mathrm{UV}$ auroras from $\sim 5$ to $\sim 50 \mathrm{kR}$. At dusk, the values are reduced to voltages of $\sim 1$ to $\sim 10 \mathrm{kV}$, energy fluxes of $\sim 0.05$ to $\sim 0.5 \mathrm{~mW} \mathrm{~m}^{-2}$, and corresponding $\mathrm{UV}$ auroras of $\sim 0.5$ to $\sim 5 \mathrm{kR}$. Overall, therefore, it can be seen that magnetosheath (cusp) plasma is too dense and too cold to need the significant accelerations required to produce significant UV aurora. On the other hand, field-aligned currents of the above strength flowing in the hot tenuous outer magnetospheric plasma require typical accelerations of a few $\mathrm{kV}$ to a few tens of $\mathrm{kV}$. The precipitating accelerated electrons then produce auroras of $\sim 5-50 \mathrm{kR}$ at dawn and $\sim 0.5-5 \mathrm{kR}$ at dusk, the auroras thus being typically an order of magnitude less intense at dusk than at dawn for a given width of the current layer, for the model values employed here. Although these numbers should be regarded as indicative only, we nevertheless note that they are reasonably commensurate with the values obtained from the HST image shown in Fig. 1, as given in Table 1 . The values we have obtained, however, do not generally approach the brightnesses estimated for the brightest localised features which have been observed, such as the intermittent brightening in the pre-noon sector, which peaks at $\sim 60 \mathrm{kR}$ in the image shown in Fig. 1 and $\sim 100 \mathrm{kR}$ in some of the images presented by Trauger et al. (1998). We suggest that this feature may be associated with reconnection-related dynamics, as will be briefly discussed in the next section.

\section{Flow and auroral dynamics}

Detailed discussion of the dynamics of the flows and auroras in Saturn's polar ionosphere is clearly beyond the scope of the initial considerations presented here. However, the auroral observations mentioned in the Introduction suggest considerable variability, thus prompting initial comment. If our suggestion concerning the origin of Saturn's auroras is correct, related to the solar wind interaction at the magnetopause boundary, two sources of variability may contribute. The first is the considerable variability in plasma parameters and flows observed in Voyager data on the magnetospheric side of the boundary (Richardson 1986, 1995; Richardson and Sittler, 1990), the origins of which are not yet understood. The second concerns the variability of the plasma and field on the other side of the boundary, which impinges from the solar wind. This may give rise to a variety of time-varying phenomena, associated, for example, with stream interfaces and shocks. In particular, we would expect the nature of the interaction to depend significantly on the direction of the IMF. For example, we may expect the oval to vary in size depending on the north-south component of the IMF, expanding in size with the amount of open flux when magnetopause reconnection is active for northward IMF, and contracting in size when magnetopause reconnection decreases with southward IMF, similar to the case for the Earth (e.g. Milan et al., 2003). However, the time scale for such changes at Saturn will be longer by more than an order of magnitude compared with Earth, reflecting the longer residence times of open tubes in the tail lobes, $\sim 50 \mathrm{~h}$ for Saturn as estimated here, compared with $\sim 3 \mathrm{~h}$ for Earth.

In addition to such slowly-varying features, the possibility also exists of observing more rapid dynamical features associated with bursts of reconnection, both at the magnetopause and in the tail, as are commonly observed at Earth (see, e.g. the recent review by Cowley et al., 2003a). Following similar ideas developed for the terrestrial environment by Siscoe and Huang (1985) and Cowley and Lockwood (1992), we may consider the effects on the flow and current system of bursts of dayside and tail reconnection. The perturbation flows and field-aligned currents associated with a burst of dayside reconnection (in the absence of tail reconnection) are indicated in Fig. 4a, following the general picture shown in Fig. 3. In this figure the central circular region is the region of open field lines, the solid lines with Xs representing the dayside merging gap, while the dashed line represents the adiaroic portion where the boundary moves with the flow. The plasma streamlines indicate the flows that are added by dayside reconnection to the general sub-corotational flows present in the closed and open field regions, as discussed above, and are associated with an interval in which the region of open flux is expanding, as indicated by the large arrows. It can be seen that these flows add to the flow shear on the dawn side of the open-closed field line boundary (dashed line), most particularly in the pre-noon sector, while subtracting from the shear somewhat in the dusk sector. Consequently, the upwarddirected field-aligned currents are enhanced in the pre-noon sector as shown, while being diminished in the post-noon sector. Additional downward field- aligned currents are also added in the equatorward region of the return flows. Thus, we suggest that the auroral brightening observed in the pre-noon sector in Fig. 1 and by Trauger et al. (1998) (and possibly the post-noon dimming as well) is due to this effect, and signifies on-going dayside reconnection. The time scale for the evolution of particular reconnection events is set by the time scale for the propagation of open flux tubes from the assumed equatorial reconnection sites into the near-planet tail, which is $\sim 2 \mathrm{~h}$ in the case of Saturn ( $\sim 5 \mathrm{~min}$ at Earth). Similarly, 


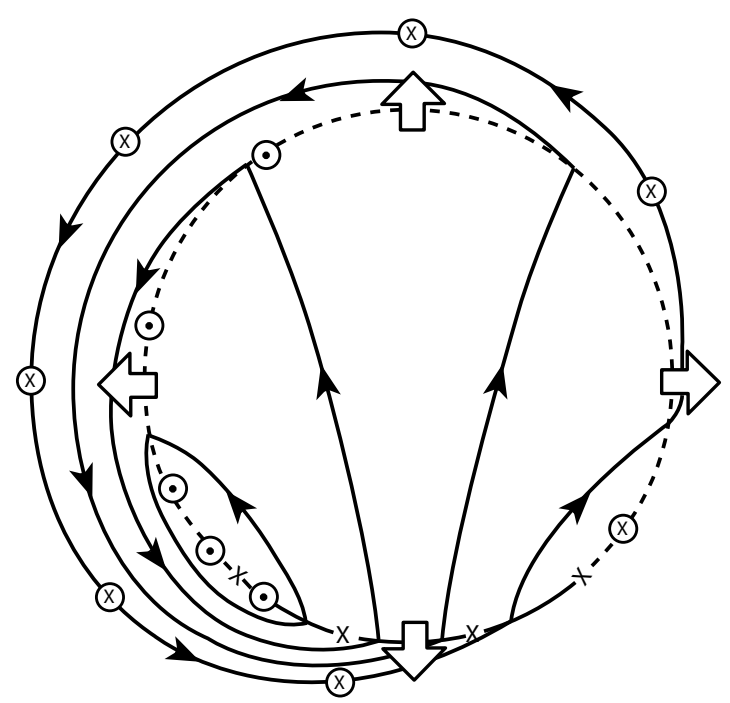

(a)

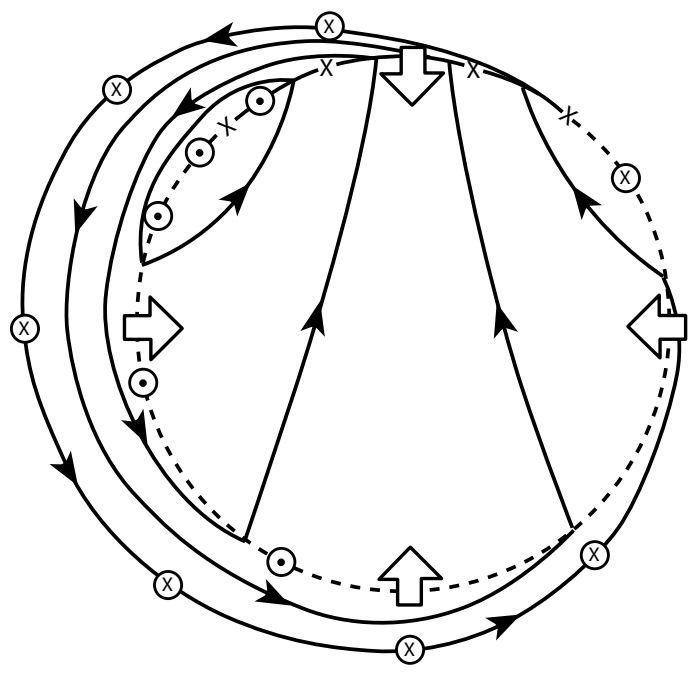

(b)

Fig. 4. Sketches showing the perturbation flows, added to the general sub-corotating flows, associated with (a) intervals of magnetopause Dungey-cycle reconnection in the absence of tail reconnection, and (b) intervals of tail Dungey-cycle reconnection in the absence of magnetopause reconnection. In each diagram the direction towards the Sun is at the bottom of the plot, dawn is to the left, and dusk to the right. The central circular region in each diagram represents the region of open field lines, where the solid line with Xs represents the "merging gap" mapping to the corresponding reconnection sites in each case, while the dashed portion represents the adiaroic region of the boundary where the boundary moves with the flow. Cases (a) and (b) correspond to an expanding and contracting polar cap, respectively, as indicated by the large arrows. The arrowed solid lines indicate plasma streamlines, while the circled dots and crosses indicate upward and downward field-aligned currents respectively.

the perturbation flows and currents associated with bursts of Dungey-cycle tail reconnection (in the absence of dayside reconnection) are shown in Fig. 4b, now associated with a contracting polar cap. These flows again enhance the upwarddirected boundary currents at dawn, and diminish them at dusk. However, the dynamic auroral signatures in this case should be centred in the post-midnight and morning sectors of the oval.

\section{Summary}

In this paper we have discussed the large-scale flow in Saturn's magnetosphere, driven by the combined action of the solar wind interaction and planetary rotation. The three components of the flow considered here are an inner region dominated by planetary rotation, where planetary plasma produced in situ from ring grains and icy moons diffuses outwards due to small-scale plasma motions, a hypothesised outer region, where planetary plasma is lost down the dusk flank tail by field-lines stretching, followed by plasmoidformation and pinching-off, as first discussed for Jupiter by Vasyliunas (1983), and finally, an outer region dominated by the solar wind interaction, driven by reconnection at the magnetopause followed by subsequent reconnection in the tail, as first discussed for Earth by Dungey (1961). Although no detailed dynamical models exist for these flow components, the flow in the dayside closed field region is constrained by thermal plasma observations obtained during the flybys of the Voyager spacecraft, showing that the dayside equatorial flow declines from near-rigid corotation close to the planet, within a few $R_{S}$, to a speed of about half of rigid corotation at $\sim 15 R_{S}$, though with some indications of a subsequent increase to perhaps three-quarters of rigid corotation in a layer a few $R_{S}$ wide adjacent to the noon magnetopause. Our discussion has employed this empirical information, and has combined it with theoretical reasoning concerning the form of the flow on open field lines, to synthesise a basic picture of the overall flow in Saturn's polar ionosphere, and hence, of the current systems as well.

In our conceptual picture, the ionosphere near-rigidly corotates between the equator and a co-latitude of $\sim 30^{\circ}$, poleward of which increasing departures from rigid corotation occur with increasing latitude, due to the requirements of transfer of angular momentum from the atmosphere to the equatorial planetary plasma. The angular velocity may then rise again somewhat in the closed-field regions bordering the polar cap occupied by the Vasyliunas-cycle flows and Dungey-cycle return flows on the dawn side, as just mentioned in relation to Voyager data, before falling to very low 
values of around a quarter of the planetary angular velocity on open field lines in the polar cap, at co-latitudes less than $\sim 15^{\circ}$. The polar cap flows consist of a slow $\left(\sim 200 \mathrm{~m} \mathrm{~s}^{-1}\right)$ anti-sunward transpolar convection driven by the Dungey cycle, together with a slow rotation which twists the tail lobe field lines, as previously discussed by Isbell et al. (1984). Such flows drive an equatorward-directed Pedersen current in the ionosphere that is proportional to the departure of the plasma angular velocity from rigid corotation, and also to the distance from the rotation axis. It also drives an eastward Hall current. However, while the Hall current largely closes in the ionosphere on latitudinal paths, the Pedersen current requires the existence of field-aligned currents related to its latitudinal variations, and hence, to the latitudinal variations of the plasma angular velocity. The pattern of flows outlined above implies a four-region pattern of field-aligned currents, with a quasi-uniform distributed downward-directed current throughout the region of open field lines, a narrow ring of upward-directed field-aligned current at the boundary of open and closed field lines, where the angular velocities abruptly change, and then further rings of downward- and upward-directed current on closed field lines associated with sub-corotation of the magnetospheric plasma. With regard to the aurora, recent work by Cowley and Bunce (2003) has shown that the latter field-aligned currents driven by sub-corotation in the region of closed field lines are too weak, and flow at too low a latitude, to explain the observed auroral luminosity. Here we have therefore examined the possibility that the auroras are instead associated with the ring of upward current which is expected to flow at the boundary of open and closed field lines, associated with the Dungey cycle. Estimates show that these currents are sufficiently intense to produce UV aurora with brightnesses between a few $\mathrm{kR}$ and a few tens of $\mathrm{kR}$, provided that they flow in a sufficiently narrow latitudinal ring, of the order of or less than $\sim 1000 \mathrm{~km}\left(1^{\circ}\right.$ latitude) wide, and are carried by hot, tenuous magnetospheric electrons (rather than cool dense magnetosheath (cusp) electrons). In this case higher $\sim 5-50 \mathrm{kR}$ brightnesses are expected at dawn, compared with $\sim 0.5-5 \mathrm{kR}$ at dusk, due to the larger expected shear in flow at the dawn boundary $\left(\sim 1550 \mathrm{~m} \mathrm{~s}^{-1}\right)$ compared with that at dusk $\left(\sim 650 \mathrm{~m} \mathrm{~s}^{-1}\right)$. We also expect the auroral oval to be displaced somewhat towards dawn, due to the magnetospheric asymmetry associated with the outflow of planetary plasma along the dusk flank magnetopause. These properties appear to be in good qualitative agreement with auroral observations obtained from spacecraft flyby data and HST images, and also accounts reasonably well for some of their quantitative parameters.

The implications of this picture are quite significant for remote studies of Saturn's system. In particular, the results imply that the bright poleward border of the auroras marks the open-closed field line boundary in the ionosphere, hence providing a remote means of investigating the variations of the magnetospheric interaction with the solar wind. In addition, we may expect to observe auroral dynamics associated with that interaction, specifically dynamic auroral fea- tures associated with episodes of reconnection at the magnetopause and in the tail. We hypothesise in particular that the variable pre-noon brightening observed in a number of HST images is associated with the upward field-aligned currents expected to be enhanced in this sector during dayside reconnection bursts.

Acknowledgements. EJB was supported during the course of this study by PPARC Grant PPA/G/O/1999/00181 and SWHC by PPARC Senior Fellowship PPA/N/S/2000/00197. RP was supported by CNRS. Part of this work is based on observations with the NASA/ESA Hubble Space Telescope, obtained at the STScI, which is operated by the AURA, Inc.

Topical Editor T. Pulkkinen thanks a referee for his help in evaluating this paper.

\section{References}

Atreya, S. K., Waite, Jr., J. H., Donahue, T. M., Nagy, A. S., and McConnell, J. C.: Theory, measurements and model of the upper atmosphere and ionosphere of Saturn, edited by Gehrels, T. and Matthews, M. S., p. 239, Univ. Arizona Press, Tucson, Arizona, USA, 1984.

Behannon, K. W., Lepping, R. P., and Ness, N. F.: Structure and dynamics of Saturn's outer magnetosphere and boundary regions, J. Geophys. Res., 88, 8791, 1983.

Broadfoot, A. L., Sandel, B. R., Shemansky, D. E., Holberg, J. B., Smith, G. R., Strobel, D. F., McConnell, J. C., Kumar, S., Hunten, D. M., Atreya, S. K., Donahue, T. M., Moos, H. W., Bertaux, J. L., Blamont, J. E., Pomphrey, R. B., and Linik, S.: Extreme ultraviolet observations from Voyager 1 encounter with Saturn, Science, 212, 206, 1981.

Bunce, E. J. and Cowley, S. W. H.: Divergence of the equatorial current in the dawn sector of Jupiter's magnetosphere: analysis of Pioneer and Voyager magnetic field data, Planet. Space Sci., 49, 1089, 2001.

Bunce, E. J. and Cowley, S. W. H.: A note on the ring current in Saturn's magnetosphere: Comparison of magnetic data obtained during the Pioneer-11 and Voyager-1 and -2 flybys, Ann. Geophysicae, 21, 661, 2003.

Bunce, E. J., Cowley, S. W. H., and Wild, J. A.: Azimuthal magnetic fields in Saturn's magnetosphere: Effects associated with plasma sub-corotation and the magnetopause-tail current system, Ann. Geophysicae, 21, 1709, 2003.

Clarke, J. T., Moos, H. W., Atreya, S. K., and Lane, A. L.: IUE detection of bursts of H Ly $\alpha$ emission from Saturn, Nature, 290, 226, 1981.

Cheng, A. F. and Waite, J. H., Jr.: Corotation lag of Saturn's magnetosphere: Global ionospheric conductivities revisited, J. Geophys. Res., 93, 4107, 1988.

Connerney, J. E. P., Acuña, M. H., and Ness, N. F.: Currents in Saturn's magnetosphere, J. Geophys. Res., 88, 8779, 1983.

Cowley, S. W. H. and Bunce, E. J.: Origin of the main auroral oval in Jupiter's coupled magnetosphere-ionosphere system, Planet. Space Sci., 49, 1067, 2001.

Cowley, S. W. H. and Bunce, E. J.: Corotation-driven magnetosphere-ionosphere coupling currents in Saturn's magnetosphere and their relation to the auroras, Ann. Geophysicae, 21, $1691,2003$. 
Cowley, S. W. H. and Lockwood, M.: Excitation and decay of solar wind-driven flows in the magnetosphere-ionosphere system, Ann. Geophysicae, 10, 103, 1992.

Cowley, S. W. H., Balogh, A. Dougherty, M. K., Dunlop, M. W., Edwards, T. M., Forsyth, R. J., Laxton, N. F., and Staines, K.: Plasma flow in the jovian magnetosphere and related magnetic effects: Ulysses observations, J. Geophys. Res., 101, 15 197, 1996.

Cowley, S. W. H., Bunce, E. J. Stallard, T. S. and Miller, S.: Jupiter's polar ionospheric flows: theoretical interpretation, Geophys. Res. Lett., 30(5), 1220, doi:10.1029/2002GL016030, 2003b.

Cowley, S. W. H., Davies, J. A., Grocott, A., Khan, H., Lester, M., McWilliams, K. A., Milan, S. E., Provan, G., Sandholt, P. E., Wild, J. A., and Yeoman, T. K.: Solar wind-magnetosphereionosphere interactions in the Earth's plasma environment, Phil. Trans. A, 361, 113, 2003a.

Cowley, S. W. H., Morelli, J. P., and Lockwood, M.: Dependence of convective flows and particle precipitation in the high-latitude dayside ionosphere on the $\mathrm{X}$ and $\mathrm{Y}$ components of the interplanetary magnetic field, J. Geophys. Res., 96, 5557, 1991.

Cowley, S. W. H., Nichols, J. D., and Bunce, E. J.: Distributions of current and auroral precipitation in Jupiter's middle magnetosphere computed from steady-state Hill-Pontius angular velocity profiles: Solutions for current sheet and dipole magnetic field models, Planet. Space Sci., 50, 717, 2002.

Desch, M. D.: Evidence for solar wind control of Saturn radio emission, J. Geophys. Res., 87, 4549, 1982.

Desch, M. D. and Rucker, H. O.: The relationship between Saturn kilometric radiation and the solar wind, J. Geophys. Res., 88, 8999, 1983.

Dungey, J. W.: Interplanetary field and the auroral zones, Phys. Rev. Lett., 6, 47, 1961.

Dungey, J. W.: The length of the magnetospheric tail, J. Geophys. Res., 70, 1753, 1965.

Galopeau, P. H. M., Zarka, P., and LeQuéau, D.: Source location of Saturn's kilometric radiation: The Kelvin-Helmholtz instability hypothesis, J. Geophys. Res., 100, 26397, 1995.

Gérard, J.-C., Dols, V., Grodent, D., Waite, J. H., Gladstone, G. R., and Prangé, R.: Simultaneous observations of the saturnian aurora and polar haze with the HST/FOC, Geophys. Res. Lett., 22, 2685, 1995 .

Gurnett, D. A., Kurth, W. S. and Scarf, F. W.: Plasma waves near Saturn: Initial results from Voyager 1, Science, 212, 235, 1981.

Hill, T. W.: Inertial limit on corotation, J. Geophys. Res., 84, 6554, 1979.

Hill, T. W.: The jovian auroral oval, J. Geophys. Res., 106, 8101, 2001.

Huang, T. S. and Hill, T. W.: Corotation lag of the jovian atmosphere, ionosphere and magnetosphere, J. Geophys. Res., 94, 3761, 1989.

Isbell, J., Dessler, A. J., and Waite, Jr., J. H., : Magnetospheric energization by interaction between planetary spin and the solar wind, J. Geophys. Res., 89, 10716, 1984.

Johnson, R. E., Pospieszalska, M. K., Sittler, Jr., E. C., Cheng, A. F., Lanzerotti, L. J., and Sieveka, E. M.: The neutral cloud and heavy ion inner torus at Saturn, Icarus, 77, 311, 1989.

Judge, D. L., Wu, F. M., and Carlson, R. W.: Ultraviolet photometer observations of the Saturnian system, Science, 207, 431, 1980.

Kaiser, M. L., Desch, M. D., Kurth, W. S., Lecacheux, A., Genova, F., Pederson, B. M., and Evans, D. R.: Saturn as a radio source, in Saturn, edited by Gehrels, T. and Matthews, M. S., p. 378,
Univ. Arizona Press, Tucson, Arizona, USA, 1984.

Knight, S.: Parallel electric fields, Planet. Space Sci., 21, 741, 1973.

Lecacheux, A. and Genova, F.: Source location of Saturn kilometric radio emission, J. Geophys. Res., 88, 8993, 1983.

Lundin, R. and Sandahl, I.: Some characteristics of the parallel electric field acceleration of electrons over discrete auroral arcs as observed from two rocket flights, in: Symposium on European Rocket Research, ESA SP-135, ESA, Noordwijk, The Netherlands, p. 125, 1978.

McGrath, M. A. and Clarke, J. T.: H I Lyman alpha emission from Saturn (1980-1990), J. Geophys. Res., 97, 13 691, 1992.

Milan, S. E., Lester, M., Cowley, S. W. H., Oksavik, K., Brittnacher, M., Greenwald, R. A., Sofko, G., and Villain, J.-P.: Variations in polar cap area during two substorm cycles, Ann. Geophysicae, 21, 1121, 2003.

Ness, N. F., Acuña, M. H., Lepping, R. P., Connerney, J. E. P., Behannon, K. W., Burlaga, L. F., and Neubauer, F. M.: Magnetic field studies by Voyager 1: Preliminary results at Saturn, Science, 212, 211, 1981.

Ness, N. F., Acuña, M. H., Behannon, K. W., Burlaga, L. F., Connerney, J. E. P., Lepping, R. P., and Neubauer, F.: Magnetic field studies by Voyager 2: Preliminary results at Saturn, Science, 215, $558,1982$.

Nichols, J. D. and Cowley, S. W. H.: Magnetosphere-ionosphere coupling currents in Jupiter's middle magnetosphere: Dependence on the effective ionospheric Pedersen conductivity and iogenic plasma mass outflow rate, Ann. Geophysicae, 21, 1419, 2003.

Pontius, Jr., D.H., : Radial mass transport and rotational dynamics, J. Geophys. Res., 102, 7137, 1997.

Pospieszalska, M. K. and Johnson, R. E.: Micrometeoritic erosion of main rings as a source of plasma in the inner Saturnian plasma torus, Icarus, 93, 45, 1991.

Rego, D., Prangé, R., and Gérard, J. C.: Lyman $\alpha$ and $\mathrm{H}_{2}$ bands from the giant planets: 1 . Excitation by proton precipitation in the jovian aurorae, J. Geophys. Res., 99, 17 075, 1994.

Reiff, P. H., Spiro, R. W., and Hill, T. W.: Dependence of polar cap potential drop on interplanetary parameters, J. Geophys. Res. 86, 7639, 1981.

Richardson, J. D.: Thermal ions at Saturn: Plasma parameters and implications, J. Geophys. Res., 91, 1381, 1986.

Richardson, J. D.: A new model for plasma transport and chemistry at Saturn, J. Geophys. Res., 97, 13 705, 1992.

Richardson, J. D.: An extended plasma model for Saturn, Geophys. Res. Lett., 22, 1177, 1995.

Richardson, J. D. and Sittler, Jr., E.C., : A plasma density model for Saturn based on Voyager observations, J. Geophys. Res., 95, 12 019, 1990.

Richardson, J. D., Eviatar, A., McGrath, M. A., and Vasyliunas, V. M.: OH in Saturn's magnetosphere: Observations and theory, J. Geophys. Res., 103, 20 245, 1998.

Sandel, B. R. and Broadfoot, A. L.: Morphology of Saturn's aurora, Nature, 292, 679, 1981.

Sandel, B. R., Shemansky, D. E., Broadfoot, A. L., Holberg, J. B., Smith, G. R., McConnell, J. C., Strobel, D. F., Atreya, S. K., Donahue, T. M., Moos, H. W., Hunten, D. M., Pomphrey, R. B., and Linik, S.: Extreme ultraviolet observations from the Voyager 2 encounter with Saturn, Science, 215, 548, 1982.

Shemansky, D. E. and Ajello, J. M.: The Saturn spectrum in the EUV: Electron excited hydrogen, J. Geophys. Res., 88, 459, 1983. 
Siscoe, G. L. and Huang, T. S.: Polar cap inflation and deflation, J. Geophys. Res., 90, 543, 1985.

Sittler, Jr., E. C., Ogilvie, K. W., and Scudder, J. D.: Survey of lowenergy plasma electrons in Saturn's magnetosphere: Voyagers 1 and 2, J. Geophys. Res., 88, 8847, 1983.

Smith, E. J., Davis, Jr., L., , Jones, D. E., Coleman, Jr., P. J., Colburn, D. S., Dyal, P., and Sonett, C. P.: Saturn's magnetosphere and its interaction with the solar wind, J. Geophys. Res., 85, $5655,1980$.

Southwood, D. J. and Kivelson, M. G.: A new perspective concerning the influence of the solar wind on Jupiter, J. Geophys. Res., 106, 6123, 2001.

Trauger, J. T., Clarke, J. T., Ballester, G. E., Evans, R. W., Burrows, C. J., Crisp, D., Gallagher III, J. S., , Griffiths, R. E., Hester, J. J., Hoessel, J. G., Holtzman, J. A., Krist, J. E., Mould, J.
R., Sahai, R., Scowen, P. A., Stapelfeldt, K. R., and Watson, A. M.: Saturn's hydrogen aurora: Wide field and planetary camera 2 imaging from the Hubble Space Telescope, J. Geophys. Res., 103, $20237,1998$.

Vasyliunas, V. M.: Plasma distribution and flow, in: Physics of the Jovian Magnetosphere, edited by Dessler, A. J., p. 395, Cambridge Univ. Press, Cambridge, UK, 1983.

Waite, Jr., J. H., Cravens, T. E., Kozyra, J., Nagy, A. F., Atreya, S. K., and Chen, R. H.: Electron precipitation and related aeronomy of the jovian thermosphere and ionosphere, J. Geophys. Res., 88, $6143,1983$.

Woch, J., Krupp, N., and Lagg, A.: Particle bursts in the jovian magnetosphere: Evidence for a near-Jupiter neutral line, Geophys. Res. Lett., 29(7), 42-1, doi:10.1029/2001GL014080, 2002. 